\title{
The Cycle Enumerator of Unimodal Permutations
}

\author{
Jean-Yves ThiBON*
}

\begin{abstract}
We give a generating function for the number of unimodal permutations with a given cycle structure.
\end{abstract}

\section{Introduction}

The problem of determining the number of unimodal permutations with a given cycle structure was proposed by Rogers [11], motivated by problems related to the structure of periodic orbits of one dimensional dynamical systems. Partial results have since been obtained by Weiss and Rogers [13] and more recently by Gannon [2]. The proofs of these results proceed by construction of explicit bijections. In this note, we propose another approach, leading to a complete solution in the form of a generating function. Our main tool is the theory of symmetric functions.

Recall that a permutation $\sigma \in \mathfrak{S}_{n}$ is said to be unimodal if for some $m \in I_{n}=$ $\{1,2, \ldots, n\}$,

$$
\sigma(1)<\sigma(2)<\ldots<\sigma(m)>\sigma(m+1)>\ldots>\sigma(n) .
$$

The set of such permutations will be denoted by $U_{n}$. For a partition $\alpha=\left(1^{a_{1}} 2^{a_{2}} \cdots n^{a_{n}}\right)$ of $n$, let $u_{\alpha}$ be the number of unimodal permutations having $\alpha$ as cycle type (i.e., having $a_{1}$ fixed points, $a_{2} 2$-cycles, etc.), and let $p_{\alpha}=p_{1}^{a_{1}} p_{2}^{a_{2}} \cdots p_{n}^{a_{n}}$ (here, the $p_{k}, k \geq 1$ are independent variables, interpreted in the sequel as power-sum symmetric functions).

Theorem 1.1 Let $c_{n}=\frac{1}{n} \sum_{d \mid n, d \text { odd }} \mu(d) 2^{n / d-1}$, where $\mu$ is the Möbius function. The cycle enumerator of unimodal permutations is given by

$$
1+2 \sum_{|\alpha| \geq 1} u_{\alpha} p_{\alpha}=\prod_{k \geq 1}\left(\frac{1+p_{k}}{1-p_{k}}\right)^{c_{k}}
$$

The coefficient of $p_{n}$ being obviously equal to $2 c_{n}$, we recover the result of Weiss and Rogers [13:

* IGM - Université de Marne-la-Vallée - Cité Descartes - 5 Boulevard Descartes — Champs-surMarne - 77454 Marne-la-Vallée cedex 2 - France - e-mail: jyt@univ-mlv.fr 
Corollary 1.2 The number of transitive unimodal permutations in $\mathfrak{S}_{n}$ is equal to $c_{n}$.

The specialization $p_{n}=t^{n}$ leads to various generating functions. The simplest example is of course the identity

$$
\prod_{k \geq 1}\left(\frac{1+t^{k}}{1-t^{k}}\right)^{c_{k}}=\frac{1}{1-2 t}
$$

which is easily checked by expanding the logarithms of both sides, and amounts to the well-known fact that $\left|U_{n}\right|=2^{n-1}$. Then, if we denote by $u_{n}^{(k)}$ the number of $\sigma \in U_{n}$ having no $k$-cycle, we find

\section{Corollary 1.3}

$$
1+2 \sum_{n \geq 1} u_{n}^{(k)} t^{n}=\left(\frac{1-t^{k}}{1+t^{k}}\right)^{c_{k}} \frac{1}{1-2 t} .
$$

For example, the number of unimodal permutations without fixed points (derangements) is seen to be $u_{n}^{(1)}=\frac{1}{3}\left(2^{n-1}+(-1)^{n}\right)$, in accordance with [2].

Finally, we note that the generating function for the number $v_{n}^{(m)}$ of unimodal permutations satifying $\sigma^{m}=1$ is a rational function:

Corollary 1.4

$$
1+2 \sum_{n \geq 1} v_{n}^{(m)} t^{n}=\prod_{d \mid m}\left(\frac{1+t^{d}}{1-t^{d}}\right)^{c_{d}} .
$$

\section{Background}

An integer $d \in\{1,2, \ldots, n-1\}$ is said to be a descent of a permutation $\sigma \in \mathfrak{S}_{n}$ if $\sigma(i)>\sigma(i+1)$. Let $\operatorname{Des}(\sigma)=D=\left\{d_{1}, d_{2}, \ldots, d_{r}\right\}$ be the descent set of $\sigma$. It is customary to encode it by a composition $I=\left(i_{1}, \ldots, i_{r+1}\right)$ of $n$ in $r+1$ parts, called the shape of $\sigma$, defined by $i_{1}=d_{1}, i_{2}=d_{2}-d_{1}, \ldots, i_{r+1}=n-d_{r}$. We write $I=C(D)=C(\sigma)$ and $D=\operatorname{Des}(I)$. These definitions are best visualized by writing $\sigma$ as a ribbon diagram, e.g., with $\sigma=42687135$,

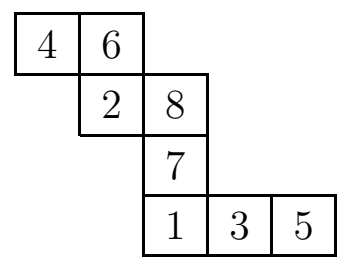

the lengths of the rows form the composition $(2,2,1,3)$ of 8 , corresponding to the descent set $\{2,4,5\}$.

Thus, unimodal permutations are those of shape $\left(n-k, 1^{k}\right)$ for some $k=0, \ldots, n-1$. The number $a_{\alpha, I}$ of permutations of shape $I$ and cycle type $\alpha$ is known to be equal to 
a scalar product of symmetric functions, which may sometimes be evaluated in closed form [5].

Let $\operatorname{Sym}=\operatorname{Sym}(X)$ be the ring of symmetric functions in the set of indeterminates $X=\left\{x_{1}, x_{2}, \ldots\right\}$, endowed with its standard scalar product for which the Schur functions $s_{\lambda}$ form an orthonormal basis (see, e.g., [9]).

Let $h_{n}=h_{n}(X)=s_{(n)}(X)$ be the complete homogeneous symmetric functions, defined by the generating series

$$
\sigma_{t}(X)=\prod_{i \geq 1}\left(1-t x_{i}\right)^{-1}=\sum_{n \geq 0} h_{n} t^{n}
$$

and $p_{n}=\sum_{i \geq 1} x_{i}^{n}$ be the power sums. We shall need the Witt symmetric functions

$$
\ell_{n}=\frac{1}{n} \sum_{d \mid n} \mu(d) p_{d}^{n / d}
$$

and

$$
L_{\alpha}=\left(h_{a_{1}} \circ \ell_{1}\right)\left(h_{a_{2}} \circ \ell_{2}\right) \cdots\left(h_{a_{m}} \circ \ell_{n}\right)
$$

where $g \circ f$ denotes the plethysm of $f$ by $g$, and the MacMahon symmetric functions (or ribbon Schur functions, denoted by $h_{I}$ in [10])

$$
r_{I}=\left|\begin{array}{cccc}
h_{i_{1}} & h_{i_{1}+i_{2}} & \cdots & h_{i_{1}+i_{2}+\cdots+i_{m}} \\
1 & h_{i_{2}} & \cdots & h_{i_{2}+\cdots+i_{m}} \\
0 & 1 & \cdots & h_{i_{3}+\cdots+i_{m}} \\
\vdots & \vdots & \ddots & \vdots \\
0 & 0 & \cdots & h_{i_{m}}
\end{array}\right|
$$

defined for any composition $I=\left(i_{1}, i_{2}, \ldots, i_{m}\right)$.

Theorem 2.1 (Gessel-Reutenauer [5]) The number $a_{\alpha, I}$ of permutations of shape I and cycle type $\alpha$ is equal to $\left\langle r_{I}, L_{\alpha}\right\rangle$.

This result was originally obtained by means of a combinatorial argument. Recently, Jöllenbeck and Reutenauer have found a simple algebraic proof, relying on the following formula, which we will also need in the sequel:

Theorem 2.2 (Scharf-Thibon [12]) Let $X$ and $Y$ be two independent sets of indeterminates, and set $\mathcal{L}(X, Y)=\sum_{\alpha} p_{\alpha}(X) L_{\alpha}(Y)$. Then,

$$
\mathcal{L}(X, Y)=\mathcal{L}(Y, X) .
$$

Theorem 1.1 is a simple consequence of the above results. The easiest way to derive it is to make use of a classical " $\lambda$-ring" trick for dealing with hook Schur functions. This works as follows. The argument $X$ of a symmetric function is identified with the formal sum of its elements: $X=x_{1}+x_{2}+\cdots$. Then, $X \cup Y$ is identified with $X+Y$, and one can define $X Y=\sum_{i, j} x_{i} y_{j}$. The power-sums behave then as ring 
homomorphisms: $p_{n}(X+Y)=p_{n}(X)+p_{n}(Y)$ and $p_{n}(X Y)=p_{n}(X) p_{n}(Y)$. Let $q$ be another indeterminate. Under the specialization $x_{k}=q^{k-1}, X$ becomes identified with the power series $(1-q)^{-1}$, with formal inverse $1-q$. The symmetric functions of $1-q$ are then specified by the condition $p_{n}(1-q)=1-q^{n}$.

With this notation, we can state the well-known identity

$$
h_{n}((1-q) X)=(1-q) \sum_{k=0}^{n-1}(-q)^{k} s_{\left(n-k, 1^{k}\right)}(X) .
$$

\section{Proof of Theorem 1.1}

Since $r_{\left(n-k, 1^{k}\right)}=s_{\left(n-k, 1^{k}\right)}$, we have by Theorem 2.1 and (1)

$$
\begin{gathered}
u_{\alpha}=\sum_{k=0}^{n-1}\left\langle s_{\left(n-k, 1^{k}\right)}, L_{\alpha}\right\rangle=\left\langle\frac{h_{n}((1-q) X)}{1-q}, L_{\alpha}(X)\right\rangle_{q=-1} \\
=\frac{1}{2}\left\langle h_{n}(X), L_{\alpha}((1-q) X)\right\rangle_{q=-1}=\left.\frac{1}{2} L_{\alpha}(1-q)\right|_{q=-1} .
\end{gathered}
$$

Therefore,

$$
1+2 \sum_{|\alpha| \geq 1} u_{\alpha} p_{\alpha}(X)=\left.\sum_{|\alpha| \geq 0} L_{\alpha}(1-q)\right|_{q=-1} p_{\alpha}(X)=\left.\sum_{|\alpha| \geq 0} p_{\alpha}(1-q)\right|_{q=-1} L_{\alpha}(X)
$$

(by Theorem 2.2). But $\left.p_{\alpha}(1-q)\right|_{q=-1}$ is zero as soon as $\alpha$ has an even part, and is equal to $2^{l(\alpha)}$ otherwise, where $l(\alpha)$ is the length of $\alpha$. Hence,

$$
\begin{aligned}
1+2 & \sum_{|\alpha| \geq 1} u_{\alpha} p_{\alpha}(X)=\sum_{\alpha \text { odd }} 2^{l(\alpha)} L_{\alpha}(X)=\sigma_{2} \circ\left[\sum_{k \geq 0} \ell_{2 k+1}(X)\right] \\
= & \exp \left\{\sum_{j \geq 1} \frac{2^{j} p_{j}}{j} \circ\left[\sum_{k \geq 0} \frac{1}{2 k+1} \sum_{d \mid k} \mu(d) p_{d}^{(2 k+1) / d}\right]\right\} \\
= & \exp \left\{\sum_{j \geq 1} \frac{2^{j} p_{j}}{j} \circ\left[\sum_{a \geq 0} \frac{\mu(2 a+1)}{2 a+1} \sum_{b \geq 0} \frac{p_{2 a+1}^{b}}{2 b+1}\right]\right\} \\
= & \exp \left\{\sum_{j \geq 1} \sum_{a \geq 0} \frac{2^{j} \mu(2 a+1)}{(2 a+1) j} \log \left(\frac{1+p_{(2 a+1) j}}{1-p_{(2 a+1) j}}\right)\right\} \\
= & \prod_{j \geq 1} \prod_{a \geq 0}\left(\frac{1+p_{(2 a+1) j}}{1-p_{(2 a+1) j}}\right)^{\frac{\mu(2 a+1) 2^{j-1}}{(2 a+1) j}}=\prod_{n \geq 1}\left(\frac{1+p_{n}}{1-p_{n}}\right)^{c_{n}}
\end{aligned}
$$




\section{Further comments}

\subsection{A $q$-analogue of Theorem 1.1}

The previous calculation can be carried out without setting $q=-1$, and this yields the enumeration of unimodal permutations according to cycle type and position of the maximum. Setting $u_{\alpha}(q)=\sum_{k} u_{\alpha, k} q^{k}$ where $u_{\alpha, k}=\left\langle s_{\left(n-k, 1^{k}\right)}, L_{\alpha}\right\rangle$ is the number of elements of $U_{n}$ with cycle type $\alpha$ and maximum at $m=n-k$, we find

$$
\begin{gathered}
1+(1-q) \sum_{n \geq 1} \sum_{|\alpha|=n} \sum_{k=0}^{n-1}(-q)^{k} u_{\alpha, k} p_{\alpha}(X)=\left\langle\sigma_{1}((1-q) Y), \mathcal{L}(Y, X)\right\rangle_{Y} \\
=\mathcal{L}(1-q, X)=\prod_{k \geq 1} \sigma_{1-q^{k}} \circ \ell_{k}(X) \\
=\exp \left\{\sum_{k \geq 1} \sum_{m \geq 1} \frac{\left(1-q^{k}\right)^{m}}{m} p_{m} \circ\left[\frac{1}{k} \sum_{d \mid k} \mu(d) p_{d}^{k / d}\right]\right\} \\
=\exp \left\{\sum_{n \geq 1} \sum_{a \geq 1} \ell_{n}\left(1-q^{a}\right) \frac{p_{n}^{a}}{a}\right\}
\end{gathered}
$$

(after some simple transformations), and setting

$$
\ell_{n}(1-q)=\frac{1}{n} \sum_{d \mid n} \mu(d)\left(1-q^{d}\right)^{n / d}=\sum_{k} a_{n k} q^{k}
$$

we obtain

\section{Theorem 4.1}

$$
\mathcal{L}(1-q, X)=\prod_{n \geq 1} \prod_{k}\left(1-q^{k} p_{n}\right)^{-a_{n k}} .
$$

The first values of the $a_{n k}$ are given by

$\ell_{1}(1-q)=1-q, \ell_{2}(1-q)=-q+q^{2}, \ell_{3}(1-q)=-q+q^{2}, \ell_{4}(1-q)=-q+2 q^{2}-q^{3}, \ldots$

so that

$$
\mathcal{L}(1-q, X)=\left(\frac{1-q p_{1}}{1-p_{1}}\right)\left(\frac{1-q p_{2}}{1-q^{2} p_{2}}\right)\left(\frac{1-q p_{1}}{1-q^{2} p_{3}}\right) \frac{\left(1-q p_{4}\right)\left(1-q^{3} p_{4}\right)}{\left(1-q^{2} p_{4}\right)^{2}} \cdots
$$

and

$$
\begin{gathered}
\sum_{|\alpha| \geq 1} u_{\alpha}(q)=\frac{1}{1-q}[\mathcal{L}(1-q, X)-1]_{q \rightarrow-q} \\
=p_{1}+p_{11}+q p_{2}+p_{111}+\left(q+q^{2}\right) p_{21}+q p_{3} \\
+p_{1111}+\left(q+q^{2}\right) p_{211}+q^{2} p_{22}+\left(q+q^{2}\right) p_{31}+\left(q+q^{2}\right) p_{4}+\cdots
\end{gathered}
$$




\subsection{Unimodal permutations and iterated brackets}

One way to generate the set of unimodal permutations is to expand the iterated Lie bracket

$$
\left[x_{1},\left[x_{2},\left[x_{3}, \cdots,\left[x_{n-1}, x_{n}\right] \cdots\right]\right]\right]=\sum_{\sigma \in U_{n}} \nu(\sigma) x_{\sigma(1)} x_{\sigma(2)} \cdots x_{\sigma(n)},
$$

where $\nu(\sigma)=(-1)^{k}$ if the descent composition of $\sigma$ is $\left(n-k, 1^{k}\right)$. Clearly, replacing the bracket by its $q$-analogue $[a, b]_{q}=a b-q b a$ leads to the same formula, with $\nu$ replaced by $\nu_{q}(\sigma)=(-q)^{k}$.

In [1], Blessenohl and Laue prove the following identity:

$$
\sum_{\sigma \in U_{n} \cap C_{\alpha}} \nu(\sigma)=\left\{\begin{array}{cc}
\alpha(d) & \text { if } \alpha=\left(d^{m}\right), \\
0 & \text { otherwise }
\end{array}\right.
$$

where $C_{\alpha}$ denotes the set of permutations of cycle type $\alpha$. This result can be easily derived from a calculation of symmetric functions. Indeed,

$$
\sum_{\sigma \in U_{n} \cap C_{\alpha}} \nu(\sigma)=\sum_{k=0}^{n-1}\left\langle s_{\left(n-k, 1^{k}\right)}, L_{\alpha}\right\rangle=\left\langle p_{n}, L_{\alpha}\right\rangle .
$$

This scalar product is non zero only when the power-sum expansion of $L_{\alpha}$ contains a term proportional to $p_{n}$, which is the case only when the product $L_{\alpha}=\prod_{i} h_{a_{i}}\left[\ell_{i}\right]$ is reduced to a single term

$$
h_{a} \circ \ell_{d}=\sum_{|\alpha|=a} \frac{1}{z_{\alpha}} p_{\alpha} \circ\left[\frac{1}{d} \sum_{j \mid d} \mu(j) p_{j}^{d / j}\right]
$$

where the term involving $p_{n}$ is

$$
\frac{p_{a}}{a} \circ\left[\frac{\mu(d)}{d} p_{d}\right]=\mu(d) \frac{p_{n}}{n},
$$

whence the result.

The most natural $q$-analogue of (身) would be the evaluation of

$$
\sum_{\sigma \in U_{n} \cap C_{\alpha}} \nu_{q}(\sigma)=\frac{1}{1-q} L_{\alpha}(1-q) .
$$

By Theorem 2.2, we see that this is precisely the content of Theorem 4.1, for

$$
\mathcal{L}(1-q, X)=\mathcal{L}(X, 1-q)=\sum_{\alpha} L_{\alpha}(1-q) p_{\alpha}(X)
$$

\subsection{Products of unimodal permutations}

The properties of unimodal permutations are directly related to quasi-symmetric functions [4] and noncommutative symmetric functions [3, 8]. To give one more example, let us remark that the result of Kreweras [7] that any unimodal permutation of $\mathfrak{S}_{n}$ is, 
in exactly $n$ ways, the product of two unimodal permutations, appears now as a special case of Corollary 5.9 of [B], describing the product $U_{n}(x) U_{n}(y)$ in the group algebra, where

$$
U_{n}(q)=\sum_{k=0}^{n-1} q^{k} \sum_{C(\sigma)=\left(n-k, 1^{k}\right)} \sigma .
$$

The coefficient of $\sigma$ in the product is $y^{r}[r+1]_{x / y}+x^{r+1}[n-r-1]_{x y}$ if $C(\sigma)=\left(n-r, 1^{r}\right)$ (unimodal case), $x^{r+s-1} y^{t+s-1}(y+1)$ if $C(\sigma)=\left(n-r-s-t, 1^{t}, s, 1^{r}\right)$ (bimodal case), and 0 otherwise (here, $\left.[n]_{q}=\left(1-q^{n}\right) /(1-q)\right)$.

\subsection{Grassmannian permutations}

All the calculations in this paper could be easily adapted to treat the case of Grassmannian permutations, i.e. permutations with at most one descent. The relevant specialization would be here $X=1+q$.

\section{References}

[1] Blessenohl D., Laue H., On the coefficients in the associative expansion of a Lie word, Europ. J. Comb. 12 (1991), 205-210.

[2] Gannon T., The cyclic structure of unimodal permutations, preprint math.DS/9906207.

[3] Gelfand I.M., Krob D., Leclerc B., Lascoux A., Retakh V.S., Thibon J.-Y., Noncommutative symmetric functions, Adv. in Math., 112, 218-348, 1995.

[4] Gessel I., Multipartite P-partitions and inner products of skew Schur functions, [in "Combinatorics and algebra", C. Greene, Ed.], Contemporary Mathematics, 34, 289-301, 1984.

[5] Gessel I., Reutenauer C., Counting permutations with given cycle structure and descent set, J. Comb. Theory A 64 (1993), 189-215.

[6] Jöllenbeck A., Reutenauer C., Eine Symmetrieeigenschaft von Solomons Algebra und der höheren Lie-Charaktere, preprint, 1999.

[7] Kreweras G., Sur les produits de permutations unimodales, C.R. Acad. Sci. Paris 291 (1980), $235-237$.

[8] Krob D., Leclerc B., Thibon J.-Y., Noncommutative symmetric functions II: Transformations of alphabets, Int. J. of Alg. and Comput. 7 (1997), 181-264.

[9] Macdonald I.G., Symmetric functions and Hall polynomials, Clarendon Press, 1995.

[10] MacMahon P.A., Combinatory Analysis, Cambridge University Press, 1915,1916; Chelsea reprint, 1960.

[11] Rogers, T.D., Remarks on Sharkovsky's theorem, Rocky Mountain J. Math. 15 (1985), 565-569.

[12] Scharf T., Thibon J.-Y., A Hopf algebra approach to inner plethysm, Adv. Math. 104 (1994), $30-58$.

[13] Weiss, A., Rogers, T.D., The number of orientation reversing cycles in the quadratic map, CMS Conference Proc., Vol. 8., Amer. Math. Soc., Providence, 1987, 703-711. 